\title{
ANÁLISE CIENCIOMÉTRICA DA FAMÍLIA BUFONIDAE GRAY, 1825 COMO FERRAMENTA PARA A CONSERVAÇÃO NO BRASIL
}

\section{SCIENTOMETRIC ANALYSIS OF THE FAMILY BUFONIDAE GRAY, 1825 AS A TOOL FOR CONSERVATION IN BRAZIL}

\author{
Caio A. Figueiredo-de-Andrade ${ }^{1 *}$, Leonardo Serafim da Silveira ${ }^{1}$ \\ ${ }^{1}$ Universidade Estadual do Norte Fluminense Darcy Ribeiro, Programa de Pós- \\ Graduação em Ciência Animal, Centro de Ciências e Tecnologias Agropecuárias, \\ Campos dos Goytacazes, Rio de Janeiro, Brasil. \\ *Rua Antônio Barbosa Neto, 563. Floresta, Salinas (MG). CEP 39560-000. Telefone: (38) \\ 9-9850-1855. E-mail: caio.herpeto@gmail.com.
}

\section{RESUMO}

A ordem Anura constitui aproximadamente $88 \%$ da biodiversidade mundial de anfíbios, sendo representada por 1039 espécies, distribuídas em 20 famílias e 90 gêneros em território brasileiro. São animais os quais apresentam elevada diversidade morfológica e comportamental e são considerados valiosos bioindicadores de qualidade ambiental, podendo ser utilizados no estudo do funcionamento de ecossistemas e delineamento de áreas prioritárias para conservação. Estudos cienciométricos permitem avaliar a produção científica em uma determinada área de interesse e, considerando a diversidade de anfíbios brasileiros aliada à importância/carência de estudos cienciométricos sobre os bufonídeos, o presente trabalho objetivou realizar um estudo quantitativo e qualitativo da produção científica sobre a família Bufonidae no Brasil. Realizou-se o levantamento bibliográfico pesquisando-se bancos de dados virtuais e foram selecionados os trabalhos publicados ao longo dos últimos 30 anos, os quais foram analisados quanto ao ano de publicação, tema da pesquisa, bioma, espécies citadas e realização em Unidades de Conservação. Foram encontrados 99 artigos, publicados entre os anos de 1986 e 2015. Os temas riqueza/diversidade e distribuição geográfica de espécies foram os mais abordados, e a maioria dos trabalhos foi realizado na Mata Atlântica. Foram citados 7 gêneros e 46 espécies, sendo o gênero Rhinella o mais estudado e Oreophrynella o menos estudado. Observou-se também que menos de $30 \%$ dos trabalhos foram realizados em áreas protegidas. Destaca-se a importância da realização de mais trabalhos em temas/ áreas onde esta família ainda não é estudada, para melhor delineamento e planejamento de atividades conservacionistas.

Palavras-chave: Amphibia; Anura; Biodiversidade; Cienciometria.

\begin{abstract}
The Anura order constitutes c.a. $88 \%$ of the world amphibian biodiversity, being represented by 1039 species, distributed along 20 families and 90 genera in Brazil. They are animals that present high morphological and behavioural diversity and are considered valuable bioindicators for environmental quality, being used on researches on ecosystems dynamics and choices of priority areas for conservation. Scientometric studies can be used to evaluate scientific production on concerning areas, and, regarding the Brazilian amphibian diversity, allied to the importance/lack of scientometric studies about the family Bufonidae, the present work aimed to quantify and qualify scientific production about this family in Brazil. Literature review was done using virtual databases, selecting works published along the past 30 years, which were analyzed on year of publication, research theme, biome, cited species and accomplishment in Conservation Unities. Ninety-nine works were found, published between 1986 and 2015; species richness/diversity and geographic distribution were the most approached
\end{abstract}


themes, and most of researches were done on the Atlantic Forest biome. Seven genera and 46 species were cited; Rhinella and Oreophrynella were the most and the less studied genera, respectively. Less than $30 \%$ of the studies were performed at protected areas. The importance of doing more research on themes/areas where this family still unstudied must be highlighted, for a better planning on conservation activities.

Keywords: Amphibia; Anura; Biodiversity; Scientometry.

\section{INTRODUÇÃO}

Dentre as ordens atuais de anfíbios, a ordem Anura apresenta a maior diversidade de espécies no mundo, representando quase $88 \%$ da riqueza de anfíbios conhecida (FROST, 2017). Essa diversidade, ocorrente no Brasil, é considerada uma das maiores do mundo, atualmente composta por 1080 espécies (SEGALLA et al., 2016), das quais 1039 são espécies de anfíbios anuros distribuídas em 20 famílias e 90 gêneros (SEGALLA et al., 2016).

Anuros são animais que apresentam uma grande variedade de formas, cores, comportamentos e modos reprodutivos (UETANABARO et al., 2008), além do grande repertório vocal emitido pelos machos, principalmente para atrair fêmeas durante o período reprodutivo (IZECKSOHN \& CARVALHO-E-SILVA, 2010).

Características como dependência da água para a reprodução (com fase larval aquática, observada na maioria das espécies), tamanho corpóreo reduzido, ectotermia e alta permeabilidade cutânea tornam os anfíbios muito sensíveis a alterações ambientais (WELLS, 2007), tais fatores permitem citá-los como valiosos bioindicadores de qualidade ambiental (LIPS et al., 2001). Além dessas características, esses animais podem ser utilizados como modelo no estudo do funcionamento de ecossistemas, por ocuparem importante posição na cadeia trófica, seja atuando como predadores ou como presas (DUELLMAN \& TRUEB, 1994; SÃO-PEDRO et al., 2008).

Os anfíbios vêm ganhando destaque mundial devido ao declínio de populações em várias regiões do mundo (HOULAHAN et al., 2000; UETANABARO et al., 2008). Fatores como a perda de hábitat, interações entre espécies, flutuações populacionais ou amostragem insuficiente são apontados como possíveis causas para o registro do declínio de populações (ETEROVICK et al., 2005; BECKER et al., 2007), além da fragmentação de habitat (BECKER et al., 2007).
SILVANO \& SEGALLA (2005) ressaltam a importância da realização de estudos regionais sobre anfíbios com o objetivo de descobrir aspectos de sua distribuição geográfica, história natural, ecologia e grau de conservação, pois ainda existe uma grande lacuna de conhecimentos básicos para grande parte da anurofauna brasileira.

A família Bufonidae Gray, 1825 encontra-se atualmente representada por 595 espécies de anfíbios anuros, apresentando distribuição geográfica cosmopolita (exceto Austrália, Madagascar e ilhas em regiões oceânicas) (FROST, 2017). Composta por animais conhecidos popularmente como "sapos" ou "cururus", essa família compreende 35 gêneros viventes (PYRON \& WIENS, 2011), dos quais oito gêneros ocorrem em território brasileiro (totalizando 85 espécies) (SEGALLA et al., 2016).

Das 15 espécies de anfíbios classificadas como ameaçadas de extinção no Livro Vermelho da Fauna Brasileira Ameaçada de Extinção (HADDAD, 2008), duas pertencem à família Bufonidae, e são do gênero Melanophryniscus Gallardo, 1961. O bufonídeo Frostius pernambucensis (Bokermann, 1962) figura dentre as 48 espécies sugeridas como ameaçadas de extinção por especialistas durante consulta ampla para a redação da Lista Oficial Brasileira de Anfíbios Ameaçados (HADDAD, 2008), contudo, não consta como espécie ameaçada na lista oficial. Vale ressaltar que cerca de $13 \%$ das espécies de anfíbios brasileiros são classificadas como "deficientes de dados" e poderiam se enquadrar em alguma categoria de ameaça de acordo com a União Internacional para Conservação da Natureza - IUCN (HADDAD, 2008).

Ao longo dos últimos anos, observa-se um aumento do interesse no monitoramento da produção científica, evidenciando a necessidade e importância da realização de estudos métricos (quantitativos e/ou qualitativos) (CAMPOS et al., 2014). Nesse panorama, a cienciometria tem ganhado certo destaque entre os métodos aplicados para avaliação da ciência, sendo 
definida como a área voltada aos estudos quantitativos e qualitativos das atividades científicas realizadas, bem como à produção, divulgação e uso das informações científicas geradas (SPINAK, 1998).

Considerando a grande diversidade de anfíbios brasileiros, sua importância ecológica, os declínios populacionais registrados e tendo em vista a importância/carência de estudos cienciométricos sobre os bufonídeos brasileiros, o presente trabalho objetivou realizar um estudo quantitativo e qualitativo da produção científica sobre a família Bufonidae no Brasil, com o intuito da compilação de dados, os quais podem ser utilizados para a tomada de decisões e ações inerentes à conservação de espécies.

\section{MATERIAL E MÉTODO}

A obtenção dos artigos foi realizada por meio do levantamento dos bancos de dados dos websites das bibliotecas SciELO (Scientific Electronic Library Online) e BHL (Biodiversity Heritage Library) e, também, do periódico Check List (Journal of species lists and distribution). Foram utilizados como descritores de busca os conjuntos de termos "Bufonidae" e
"Brazil", e também "Bufonidae" e "Brasil", objetivando abranger as pesquisas desenvolvidas com a família Bufonidae (Amphibia: Anura) em território brasileiro.

Foram utilizados os artigos publicados ao longo dos últimos 30 anos encontrados nas buscas, os quais foram analisados quanto ao ano de publicação (em períodos de 5 anos), tema da pesquisa (anatomia/morfologia; bioacústica; conservação; decompositores; dieta/ forrageamento; dinâmica populacional; distribuição geográfica; etologia; fisiologia; novas espécies/taxonomia; parasitologia; reprodução e riqueza/diversidade), bioma (Caatinga; Cerrado; Amazônia; Mata Atlântica; Pampas e Pantanal), espécies citadas e realização ou não em Unidades de Conservação.

A nomenclatura aqui utilizada foi atualizada, seguindo SEGALLA et al. (2016) e FROST (2017).

\section{Resultados e discussão}

A busca combinada de publicações resultou em um total de 99 artigos publicados nos últimos 30 anos. Destes, $97 \%$ foram publicados nos últimos 15 anos (Figura 1).

Figura 1. Produção científica abordando bufonídeos brasileiros nos últimos 30 anos.

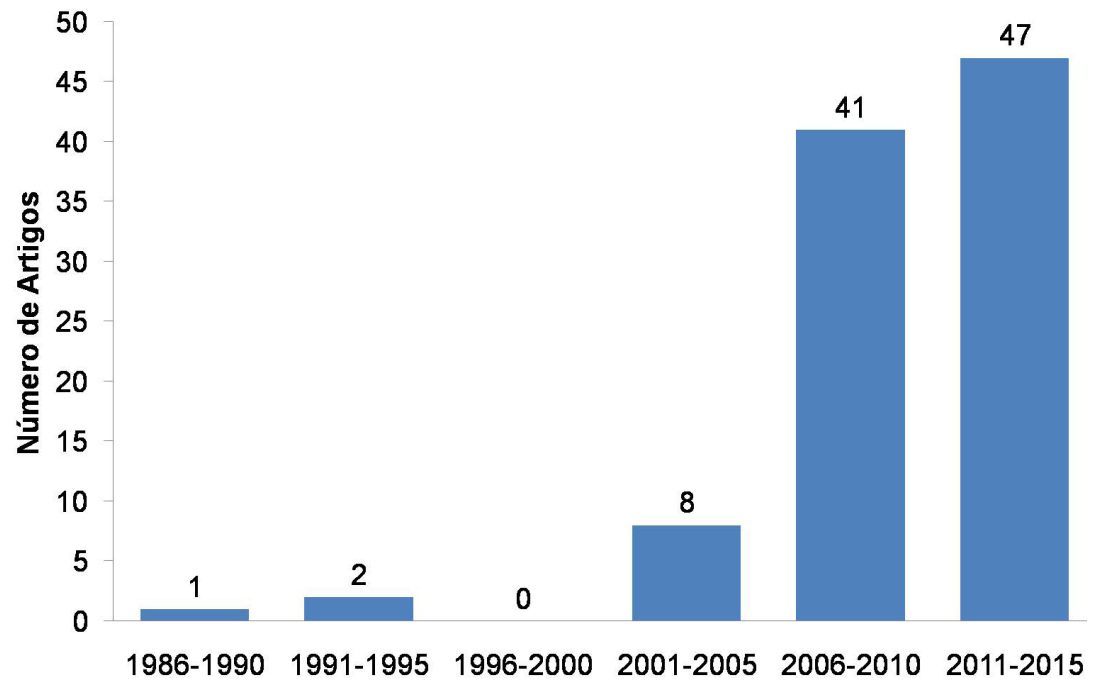

Tal resultado condiz com a evolução da história da herpetologia brasileira, uma vez que, no início da década de 80 , não existiam muitos herpetólogos brasileiros com vasta experiência na área (NASCIMENTO \& OLIVEIRA, 2007). Com o passar dos anos, esses especialistas formaram orientados de graduação e pós-graduação ainda na década de 80 e, no início dos anos 90, os quais hoje formam ou formaram um maior número de herpetólogos (NASCIMENTO \& OLIVEIRA,
2007), refletindo em uma maior quantidade de publicações nos últimos anos.

Dos artigos analisados, $60 \%$ abordaram os temas riqueza/diversidade e Distribuição Geográfica de espécies. Cada um dos demais temas totalizou menos de $8 \%$ das abordagens (Figura 2). Os artigos os quais abordaram mais de um tema foram contabilizados mais de uma vez. 
Figura 2. Distribuição dos artigos abordando bufonídeos brasileiros nos últimos 30 anos, separados por diferentes temas.

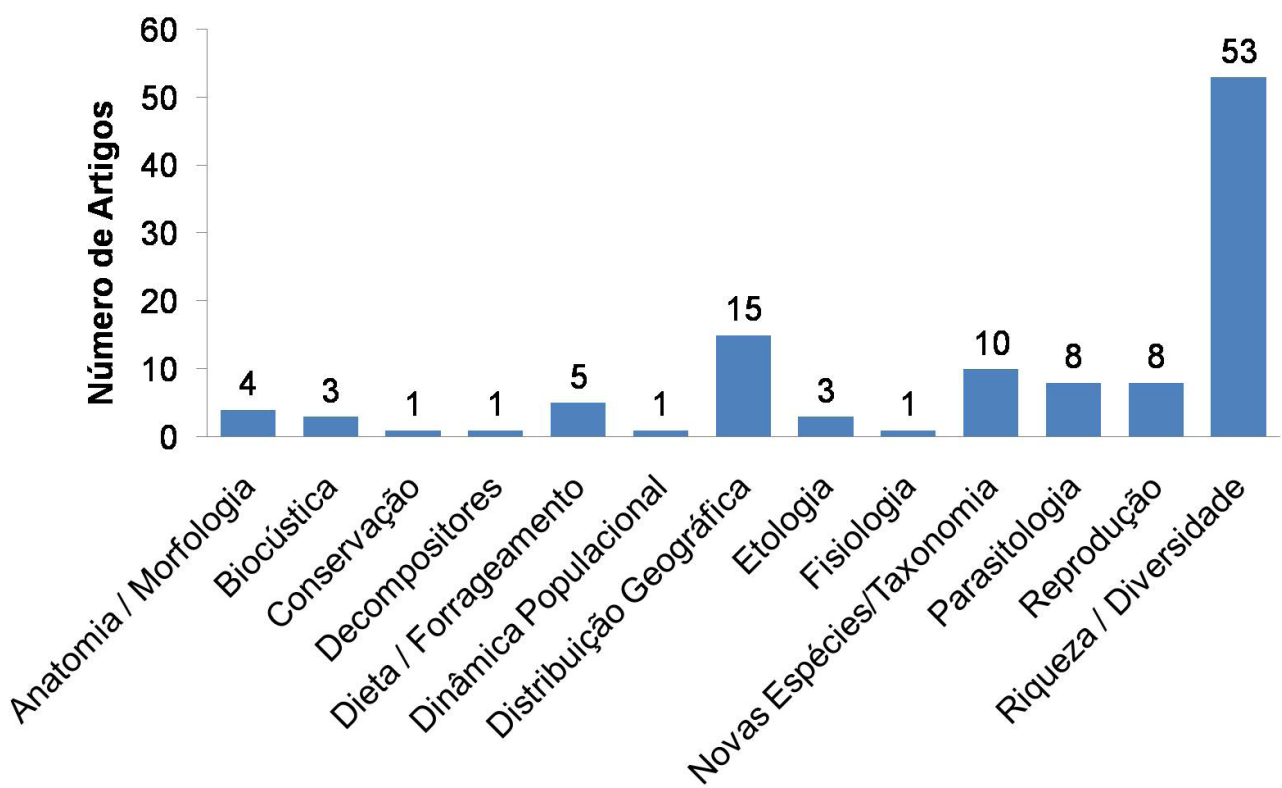

Em um país de proporções geográficas tão grandes quanto o Brasil, espera-se que boa parte de seu patrimônio natural esteja em uma fase não tão avançada de reconhecimento, ressaltando a importância da realização dos trabalhos relacionados ao inventário de espécies e conhecimento da biodiversidade de determinados ambientes. Dessa forma, acredita-se que a realização dos trabalhos relacionados aos temas riqueza/ diversidade e distribuição geográfica de espécies tem sua devida importância no momento atual, conforme o proposto por SILVANO \& SEGALLA (2005). À medida que o conhecimento da riqueza da anurofauna brasileira aumente, espera-se que trabalhos com temas mais específicos (e.g. dinâmica populacional, bioacústica, reprodução) venham a ser realizados.

De acordo com a atual classificação dos ambientes terrestres, seis biomas brasileiros podem ser identificados: Mata Atlântica, Amazônia, Cerrado, Caatinga, Pantanal e Pampas (IBGE, 2004). A maioria dos trabalhos analisados foi realizada na Mata Atlântica (57\%), seguida pelos trabalhos realizados na Amazônia e no Cerrado (18\% cada); trabalhos realizados na Caatinga e no Pantanal totalizaram apenas 5\% e 3\%, respectivamente, e nenhum trabalho foi realizado nos Pampas (Figura 3).

Figura 3. Distribuição dos artigos abordando bufonídeos brasileiros nos últimos 30 anos, separados por diferentes biomas.

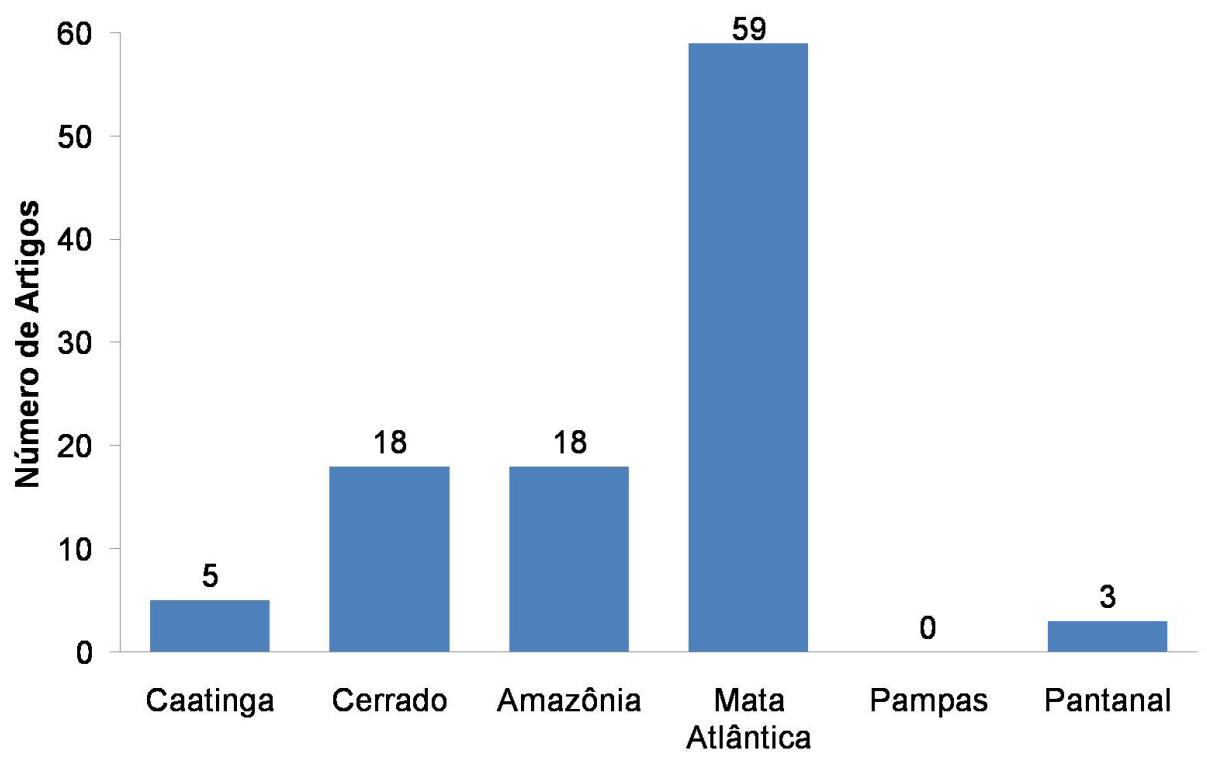


Analisando a localização das instituições de pesquisa às quais os autores estariam filiados, observou-se que quase $50 \%$ delas situam-se na região Sudeste do Brasil, fator que pode ser associado a maior concentração de trabalhos realizados na Mata Atlântica (por questões de logística, espera-se que as instituições realizem suas pesquisas em ambientes próximos à sua localização geográfica) (HADDAD, 2008). O alto grau de alterações antrópicas realizado na Mata Atlântica (atualmente restam apenas $11,73 \%$ da cobertura original desse bioma, segundo RIBEIRO et al. (2009)) e o fato de ser considerada um dos hotspots mundiais de biodiversidade (MYERS et al., 2000) também justificam esse grande esforço em compreender melhor sua biodiversidade. Contudo, vale ressaltar o aumento no interesse na biodiversidade de outros biomas brasileiros, uma vez que um número crescente de trabalhos vem sendo desenvolvidos em ambientes de Amazônia,
Cerrado (outro hotspot de biodiversidade, de acordo com MYERS et al. (2000)) e Caatinga nas últimas duas décadas.

Foram citados sete gêneros e 46 espécies nos 99 artigos analisados no presente trabalho (Tabela 1). Os artigos abordaram uma quantidade significativa de táxons, pois compreenderam quase $88 \%$ dos gêneros e $55 \%$ das espécies registradas no Brasil (SEGALLA et al., 2016). As espécies pertencentes ao gênero Rhinella Fitzinger, 1826 foram as mais citadas (quase $77 \%$ das citações), sendo Rhinella schneideri (Werner, 1894) citada 24 vezes, Rhinella icterica (Spix, 1824) 18 vezes e Rhinella ornata (Spix, 1824) 17 vezes. O gênero Oreophrynella Boulenger, 1895 foi o menos citado, apenas uma única vez, no trabalho que descreve a espécie Oreophrynella weiassipuensis Señaris, Nascimento \& Villarreal, 2005, coletada em um ambiente na fronteira do Brasil com a Guiana.

Tabela 1. Número de citações para cada espécie nos artigos analisados.

\begin{tabular}{|c|c|}
\hline Espécie & $N^{0}$ de Citações \\
\hline Amazophrynella bokermanni (Izecksohn, 1994 “1993”) & 2 \\
\hline Amazophrynella minuta (Melin, 1941) & 4 \\
\hline Atelopus spumarius Cope, 1871 & 3 \\
\hline Dendrophryniscus berthalutzae Izecksohn, 1994 “1993” & 2 \\
\hline Dendrophryniscus brevipollicatus Jiménez De La Espada, 1871 “1870” & 8 \\
\hline Dendrophryniscus carvalhoi Izecksohn, 1994 “1993” & 2 \\
\hline Dendrophryniscus leucomystax Izecksohn, 1968 & 1 \\
\hline Dendrophryniscus proboscideus (Boulenger, 1882) & 1 \\
\hline Dendrophryniscus skuki (Caramaschi, 2012) & 1 \\
\hline Melanophryniscus devincenzii Klappenbach, 1968 & 3 \\
\hline Melanophryniscus dorsalis (Mertens, 1933) & 2 \\
\hline Melanophryniscus fulvoguttatus (Mertens, 1937) & 1 \\
\hline Melanophryniscus moreirae (Miranda-Ribeiro, 1920) & 2 \\
\hline Melanophryniscus setiba Peloso, Faivovich, Grant, Gasparini \& Haddad, 2012 & 1 \\
\hline Melanophryniscus simplex Caramaschi \& Cruz, 2002 & 1 \\
\hline Melanophryniscus sp. nov. * & 1 \\
\hline Melanophryniscus spectabilis Caramaschi \& Cruz, 2002 & 1 \\
\hline Oreophrynella weiassipuensis Señaris, Nascimento \& Villarreal, 2005 & 1 \\
\hline Rhaebo guttatus (Schneider, 1799) & 7 \\
\hline Rhinella abei (Baldissera-Jr,Caramaschi \& Haddad, 2004) & 1 \\
\hline Rhinella achavali (Maneyro, Arrieta \& De Sá, 2004) & 1 \\
\hline Rhinella arenarum (Hensel, 1867) & 2 \\
\hline
\end{tabular}




\section{Conclusão}

\begin{tabular}{|c|c|}
\hline Espécie & $\mathbf{N}^{0}$ de Citações \\
\hline Rhinella bergi (Céspedez, 2000 “1999”) & 1 \\
\hline Rhinella castaneotica (Caldwell, 1991) & 1 \\
\hline Rhinella cerradensis Maciel, Brandão, Campos \& Sebben, 2007 & 2 \\
\hline Rhinella crucifer (Wied-Neuwied, 1821) & 8 \\
\hline Rhinella dorbignyi (Duméril \& Bibron, 1841) & 1 \\
\hline Rhinella fernandezae (Gallardo, 1957) & 3 \\
\hline Rhinella granulosa (Spix, 1824) & 13 \\
\hline Rhinella henseli (A. Lutz, 1934) & 1 \\
\hline Rhinella hoogmoedi Caramaschi \& Pombal, 2006 & 6 \\
\hline Rhinella icterica (Spix, 1824) & 18 \\
\hline Rhinella inopina Vaz-Silva, Valdujo \& Pombal, 2012 & 1 \\
\hline Rhinella jimi (Stevaux, 2002) & 6 \\
\hline Rhinella major (Muller \& Helmich, 1936) & 2 \\
\hline Rhinella margaritifera (Laurenti, 1768 ) & 10 \\
\hline Rhinella marina (Linnaeus, 1758) & 12 \\
\hline Rhinella mirandaribeiroi (Gallardo, 1965) & 2 \\
\hline Rhinella ocellata (Günther, 1859 “1858”) & 3 \\
\hline Rhinella ornata (Spix, 1824) & 17 \\
\hline Rhinella proboscidea (Spix, 1824) & 1 \\
\hline Rhinella pygmaea (Myers \& Carvalho, 1952) & 5 \\
\hline Rhinella rubescens (A. Lutz, 1925) & 3 \\
\hline Rhinella schneideri (Werner, 1894) & 24 \\
\hline Rhinella scitula (Caramaschi \& Niemeyer, 2003) & 1 \\
\hline Rhinella veredas (Brandão,Maciel \& Sebben, 2007) & 2 \\
\hline
\end{tabular}

* Citado em ALMEIDA et al. (2011)

As espécies do gênero Rhinella representam cerca de $47 \%$ do total de espécies de bufonídeos brasileiros (SEGALLA et al., 2016), muitas dessas apresentando ampla distribuição geográfica ( $R$. schneideri ocorre em quase todos os biomas brasileiros, com exceção da Amazônia e Pampas, por exemplo). A grande diversidade de espécies desse gênero, associada à sua ampla distribuição geográfica, é apontada como principal motivo para o elevado número de citações observadas no presente trabalho.

Em contrapartida, o gênero Oreophrynella representa apenas $2,3 \%$ do total de espécies de bufonídeos brasileiros (SEGALLA et al., 2016), com distribuição restrita ao bioma Amazônico, em local de difícil acesso. A pequena diversidade de espécies desse gênero, associada à sua restrita distribuição geográfica, é apontada como principal motivo para um número tão pequeno de citações.

Dos artigos analisados, 27 relataram ter suas atividades realizadas em algum tipo de área protegida, sendo 17 destes realizados em Unidades de Conservação reconhecidas pelo Sistema Nacional de Unidades de Conservação (15 em Unidades de Proteção Integral e 2 em Unidades de Uso Sustentável) (Figura 4). Uma vez que menos de $30 \%$ dos trabalhos analisados foram realizados em áreas protegidas, ressalta-se a importância da implementação de mais áreas destinadas à conservação e de conhecer melhor a riqueza de áreas protegidas, pois a obtenção de conhecimento sobre o que deve ser preservado é tão importante quanto a preservação em si (IVANISSEVICH, 2007). 
Figura 4. Distribuição dos artigos abordando bufonídeos brasileiros nos últimos 30 anos, realizados em áreas protegidas.

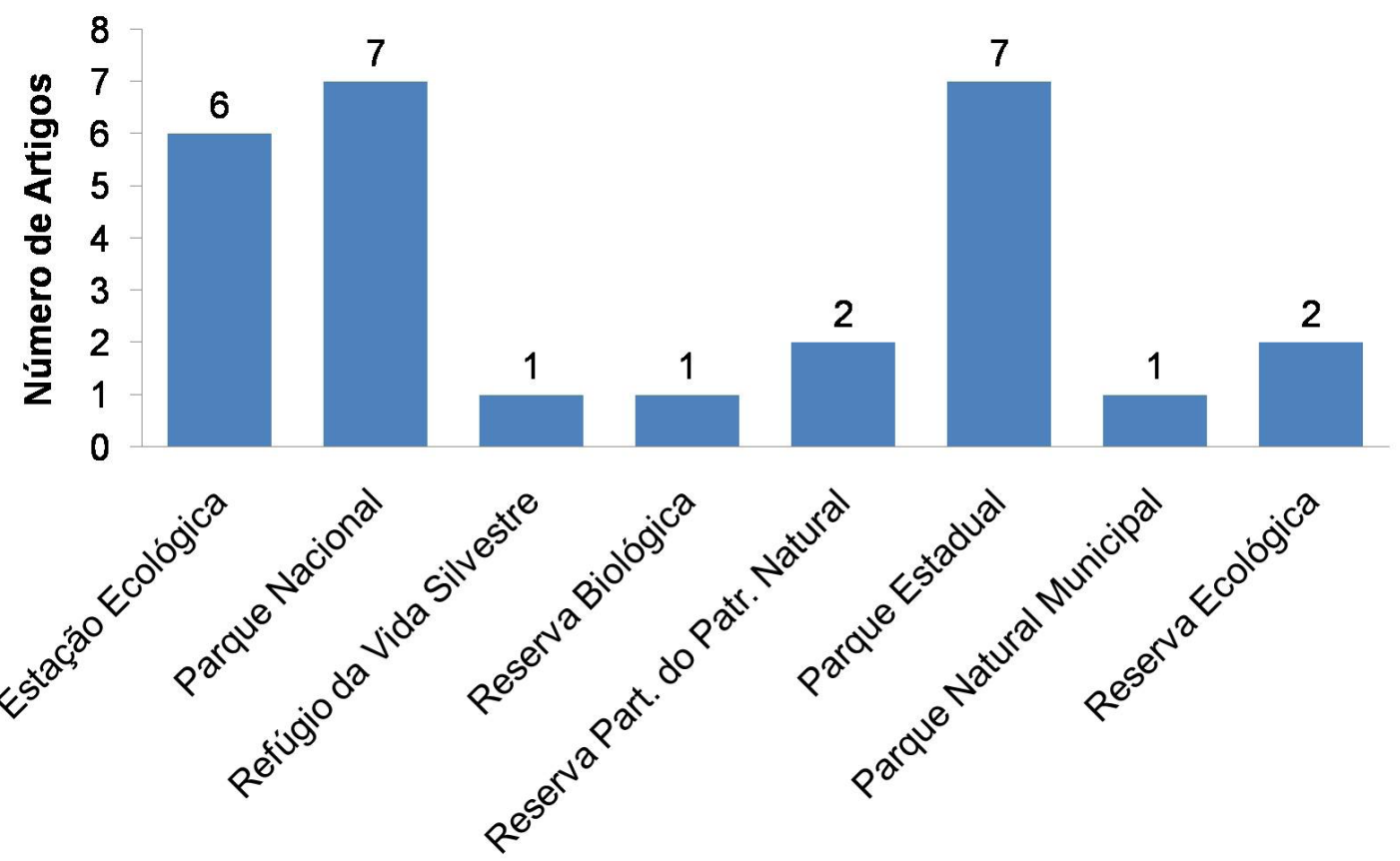

\section{CONCLUSÕES}

Nota-se um crescimento do interesse na realização de pesquisas envolvendo bufonídeos brasileiros ao longo dos anos, e espera-se um número crescente de trabalhos com temas mais específicos para os próximos anos, porque os temas riqueza/diversidade e distribuição geográfica de espécies tendem a ser menos abordados.

Salienta-se a necessidade de obtenção de conhecimento sobre a biodiversidade de outros biomas brasileiros, especialmente o Cerrado - por ser considerado um hotspot de biodiversidade mundial, Caatinga, Pantanal e Pampas - devido ao pequeno montante de pesquisas realizadas nesses ambientes até o presente momento.

Espécies mais comuns e com ampla distribuição geográfica foram as mais citadas nos trabalhos analisados, enfatizando a necessidade de obter-se mais conhecimento sobre espécies endêmicas, ou com distribuição geográfica restrita. Mais trabalhos sobre dinâmica populacional devem ser desenvolvidos nos próximos anos, com o propósito de compreender melhor padrões de flutuação populacional em anfíbios, sejam estas causadas por fenômenos naturais ou por atividade antrópica.
A obtenção de conhecimento, relacionada à biodiversidade contida em Unidades de Conservação e áreas protegidas, deve ser prioritária, para que decisões corretas de manejo, visando à conservação e preservação ambiental, sejam tomadas.

Menos de 30\% dos trabalhos analisados foram realizados em áreas protegidas, destacando a importância da realização de mais trabalhos em áreas destinadas à preservação ambiental.

\section{AGRADECIMENTOS}

Agradecemos aos professores Diego J. Santana, Sergio Potsch de Carvalho e Silva e Ana Maria Paulino Telles de Carvalho e Silva pelas sugestões e revisões iniciais do manuscrito. Ao professor André Canela Brito Nobre pela revisão de línguas. À Coordenação de Aperfeiçoamento de Pessoal de Nível Superior pela bolsa de estudos concedida.

\section{REFERÊNCIAS}

ALMEIDA, A. P.; GASPARINI, J. L.; PELOSO, P. L. V. Frogs of the state of Espírito Santo, southeastern Brazil The need for looking at the 'coldspots'. Checklist, v.7, n.4, p. 542-560, 2011.

BECKER, C. G.; FONSECA, C. R.; HADDAD, C. F. B.; BATISTA, R. F.; PRADO, P. I. Habitat Split and the Global Decline of Amphibians. Science, v.318, p. 1775-1777, 2007.

CAMPOS, F. S.; BRITO, D.; SOLÉ, M. Diversity patterns, research trends and mismatches of the investigative efforts 
to amphibian conservation in Brazil. Anais da Academia Brasileira de Ciências, v.86, n.4, p. 1873-1886, 2014.

DUELLMAN, W. E.; TRUEB, L. Biology of Amphibians. New York: McGraw Hill, 670p, 1994.

ETEROVICK, P. C.; CARNAVAL, A. C. O. Q.; BORGESNOJOSA, D. M.; SILVANO, D. L. \& SEGALLA, M. V. Amphibian Declines in Brazil: An Overview. Biotropica, v.37, n.2, p. 166-179, 2005.

FROST, D. R. Amphibian Species of the World: an Online Reference. Version 6.0. Disponível em: http://research. amnh.org/herpetology/amphibia/index.html. American Museum of Natural History, New York, USA. Acessado em 12 de abril de 2017, 2017.

HADDAD, C. F. B. Uma Análise da Lista Brasileira de Anfíbios Ameaçados de Extinção. In: Livro vermelho da fauna brasileira ameaçada de extinção (Machado, A.B.M., Drummond, G.M.M. \& Paglia, A.P., Eds. MMA, Brasília, DF, Fundação Biodiversitas, Belo Horizonte, MG, p. 287-320, 2008.

HOULAHAN, J. E.; FINDLAY, C. S.; SCHMIDT, B. R.; MEYER, A. H.; KUZMIN, S. L. Quantitative evidence for global amphibian population declines. Nature, v.404, p. 752-755, 2000.

IBGE. Mapa de Biomas e de Vegetação. Disponível em http://www.ibge.gov.br/. Instituto Brasileiro de Geografia e Estatística. Acessado em 20 de janeiro de 2017, 2004.

IVANISSEVICH, A. Conhecer para preservar. Cobertura jornalística feita a partir de conferências e mesas-redondas apresentadas na 59 ${ }^{\mathrm{a}}$ Reunião Anual da Sociedade Brasileira para o Progresso da Ciência (SBPC). Imprinta Express, Belém, 76p, 2007.

IZECKSOHN, E.; CARVALHO-E-SILVA, S. P. Anfíbios do Município do Rio de Janeiro, $2^{\mathrm{a}}$ ed. Editora UFRJ, $158 \mathrm{p}, 2010$.

LIPS, K. R.; REASER, J. K.; YOUNG, B. E.; IBÁÑEZ, R. Monitoreo de anfibios en América Latina: Manual de protocolos. Society for the study of amphibians and reptiles, Herpetological Circular, n.30, 115p, 2001.

MYERS, N.; MITTERMEIER, R. A.; MITTERMEIER, C. G.; DA FONSECA, G. A. B.; KENT, J. Biodiversity hotspots for conservation priorities. Nature, v.403, p. 853$858,2000$.

NASCIMENTO, L. B.; OLIVEIRA, M. E. Herpetologia no Brasil II. Sociedade Brasileira de Herpetologia, 354p, 2007.

PYRON, R. A.; WIENS, J. J. A large-scale phylogeny of Amphibia including over 2800 species, and revised classification of extant frogs, salamanders, and caecilians. Molecular Phylogenetics and Evolution, v.61, p. 543-583, 2011.
RIBEIRO, M. C.; METZGER, J. P.; MARTENSEN, A. C.; PONZONI, F. J.; HIROTA, M. M. The Brazilian Atlantic Forest: How much is left, and how is the remaining forest distributed? Implications for conservation. Biological Conservation, v.142, p. 1141-1153, 2009.

SÃO-PEDRO, V. A.; MOURA, M. R.; FEIO, R. N. Os Anfíbios e a Serra do Ouro Branco - Minas Gerais. Viçosa: UFV, Belo Horizonte: FAPEMIG, 16p, 2008.

SEGALlA, M. V.; CARAMASCHI, U.; CRUZ, C. A. G.; GRANT, T.; HADDAD, C. F. B; GARCIA, P. C. A.; BERNECK, B. V. M.; LANGONE, J. A. Brazilian Amphibians: List of Species. Herpetologia Brasileira, v.5, n.2, p. 34-46, 2016.

SILVANO, D. L.; SEGALlA, M. V. Conservação de anfíbios no Brasil. Megadiversidade, v.1, n.1, p. 79-86, 2005.

SPINAK, E. Indicadores cienciométricos. Ciência da Informação, v.27, n.2, p. 141-148, 1998.

UETANABARO, M.; PRADO, C. P. A.; RODRIGUES, D. J.; GORDO, M.; CAMPOS, Z. Guia de Campos dos Anuros do Pantanal e Planaltos de Entorno $=$ Field guide to the anurans of the Pantanal and surrounding Cerrados. Editora UFMS, 196p, 2008.

WELLS, K. D. The Ecology and Behavior of Amphibians. Chicago and London: The University of Chicago Press, 1148 p, 2007. 